\title{
Multi-H: Efficient Recovery of Tangent Planes in Stereo Images
}

\author{
Daniel Barath, Levente Hajder \\ \{barath.daniel,hajder.levente\}@sztaki.mta.hu \\ Jiri Matas \\ matas@cmp.felk.cvut.cz
}

\author{
MTA SZTAKI \\ Budapest, Hungary \\ CMP, Czech Technical University \\ Prague, Czech Republic
}

Multi-H - an efficient method for the recovery of the tangent planes of a set of point correspondences satisfying the epipolar constraint is proposed. The problem is formulated as a search for a labeling minimizing an energy that includes a data and spatial regularization terms. The number of planes is controlled by a combination of Mean-Shift and $\alpha$-expansion.

The input of Multi-H are point correspondences with local affine transformations and the epipolar geometry. We use Matching On Demand with view Synthesis (MODS) method [3] since it provides accurate local affinities, the fundamental matrix $\mathbf{F}$ and point correspondences consistent with $\mathbf{F}$. Using Homography from Affine transformation and Fundamental matrix (HAF) method 1 a homography is estimated for every single correspondence.

The alternating minimization stage of the algorithm repeats:

1. Mean-Shift is applied to the density function built on the homographies to reduce the complexity of the problem and to find the modes in the homography space. This procedure assumes that many points have the same tangent plane and these planes form a mode in the space of homographies.

2. $\alpha$-expansion is applied to the correspondences assigning a label to each. A label is associated with a homography.

3. Least-squares homography re-fitting step uses the HAF method to re-estimate the homographies exploiting the labeling provided by Step 2 .

Convergence is reached when both the number of the clusters and the energy remain unchanged. It is guaranteed since the first step does not increase the number of clusters, the others decrease the energy, and the set of labeling is finite.

The speed of Multi-H was measured on two sets consisting of 100 and 500 correspondences. The processing time for the 100 and 500 correspondences were 0.04 and $0.80 \mathrm{sec}$. on a desktop PC with Intel Core i5-4690 CPU, $3.50 \mathrm{GHz}$ using 4 cores.

Test 1. - Tangent plane estimation is accurately solved by Multi-H as it refines the initial estimates by partitioning the correspondences based on the similarity of their tangents. The figure below shows the recovered surface normals coloured by their labels in two images of fountain-P11 dataset.
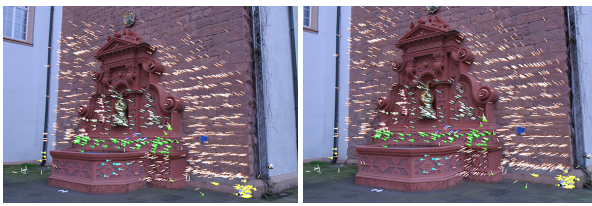

The table below shows the improvement in surface normal estimation between selected frames - angular errors in degrees.

\begin{tabular}{|c||ccc|}
\hline Frames & Affine Detector & EG- $L_{2}$-Opt & Multi-H \\
\hline $1-2$ & $35.7^{\circ}$ & $35.5^{\circ}$ & $\mathbf{1 4 . 4}^{\circ}$ \\
$1-5$ & $19.0^{\circ}$ & $16.7^{\circ}$ & $\mathbf{7 . 0}^{\circ}$ \\
$3-5$ & $24.9^{\circ}$ & $23.1^{\circ}$ & $\mathbf{9 . 0}^{\circ}$ \\
$5-9$ & $20.0^{\circ}$ & $17.8^{\circ}$ & $\mathbf{7 . 1}^{\circ}$ \\
$6-8$ & $22.5^{\circ}$ & $19.9^{\circ}$ & $\mathbf{8 . 8}^{\circ}$ \\
\hline
\end{tabular}

Test 2. - Multiple plane recovery is a long-standing problem [2]. The combination of Multi-H with a compatibility criterion $\left\|H^{T} F+F^{T} H\right\|_{F}>\theta$ leads to results superior to the state-of-the-art multi-plane fitting techniques, where $H$ is a homography, $F$ the fundamental matrix, and $\theta$ a threshold.

\begin{tabular}{|ll||rr|}
\hline & & mean & median \\
\hline J-Linkage & (ECCV 2008) & 25.50 & 24.48 \\
SA-RCM & (CVPR 2012) & 28.30 & 29.40 \\
T-Linkage & (CVPR 2014) & 24.66 & 24.53 \\
RPA & (BMVC 2015) & 17.20 & 17.78 \\
Grdy-RansaCov & (CVPR 2016) & 26.85 & 28.77 \\
ILP-RansaCov & (CVPR 2016) & 12.91 & 12.34 \\
Multi-H & (BMVC 2016) & $\mathbf{4 . 4 0}$ & $\mathbf{2 . 4 1}$ \\
\hline
\end{tabular}

The table above compares the mean and median misclassification errors on the AdelaideRMF dataset. Every algorithm, including Multi-H, has been tuned separately on each image pair to allow comparison with the literature. Results, using a fixed set-up, are shown in the table below.

\begin{tabular}{|l||cccc|}
\hline & T-Linkage & SA-RCM & RPA & Multi-H \\
\hline johnsa & 34.28 & 36.73 & 10.76 & $\mathbf{9 . 3 3}$ \\
johnsb & 24.04 & 16.46 & 26.76 & $\mathbf{1 0 . 1 4}$ \\
ladysymon & 24.67 & 39.50 & 24.67 & $\mathbf{4 . 4 9}$ \\
neem & 25.65 & 41.45 & 19.86 & $\mathbf{2 . 0 0}$ \\
old & 20.66 & 21.30 & 25.25 & $\mathbf{1 . 7 9}$ \\
sene & 7.63 & 20.20 & 0.42 & $\mathbf{0 . 0 0}$ \\
\hline mean & 22.82 & 29.27 & 17.95 & $\mathbf{4 . 7 9}$ \\
median & 24.36 & 29.02 & 22.27 & $\mathbf{3 . 7 4}$ \\
\hline
\end{tabular}

Conclusions. Multi-H is accurate, outperforms state-of-theart multi-homography fitting techniques for both fixed and perimage parameter setting. In most applications, Multi-H will run significantly faster than the affine-covariant detectors providing the input.

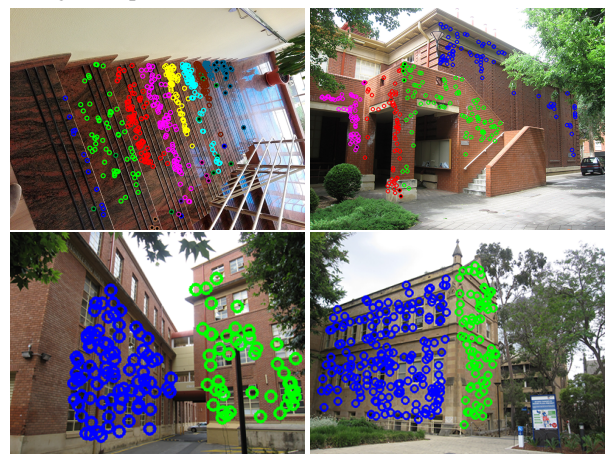

Recovered dominant planes

[1] D. Barath and L. Hajder. Novel ways to estimate homography from local affine transformations. In VISAPP, 2016.

[2] H. Isack and Y. Boykov. Energy-based geometric multimodel fitting. IJCV, 2012.

[3] D. Mishkin, J. Matas, and M. Perdoch. MODS: Fast and robust method for two-view matching. CVIU, 2015. 